\title{
Analysis of Stress and Strain Leading to Musculo Skeletal Disorders of Work Related Activities Using Finite Element Method
}

\author{
K. V. Manjunath ${ }^{1}$, Dr. B. Ravishankar ${ }^{2}$ \\ ${ }^{1}$ Research Scholar, VTU- RRC, BMS College of Engineering, Bangalore-560019, Karnataka \\ ${ }^{2}$ Professor (IEM) \& Placement Officer, BMS College of Engineering, Bangalore-560019, Karnataka
}

\begin{abstract}
Work-related musculoskeletal disorders (MSDs) are impairments of body structures such as muscles, joints, tendons, ligaments, nerves, bones or a localized blood circulation system caused or aggravated primarily by the performance of work and by the effects of the immediate environment where the work is carried out. Most work related MSDs are cumulative disorders, resulting from repeated exposures to high-or low-intensity loads over a long period of time. The symptoms may vary from discomfort and pain to decreased body function and invalidity. Although it is not clear to what extent MSDs are caused by work, their impact on working life is huge. MSDs can interfere with activities at work and can lead to reduced productivity, sickness absence and chronic occupational disability. The aim of work is to analyze the joints, using finite element method (FEM) approach with different case studies and to study on the role of ergonomics in preventing work related MSD disorders
\end{abstract}

Keywords: musculoskeletal disorders (MSDs), finite element method (FEM), Interventions, Chronic occupational disability, Exclusion Criteria

\section{Objective of Work}

The aim of this work is to study on preventing work related MSD disorders and investigate the research on the prevention of work-related MSDs. using finite element analysis, Estimation of static linear analysis and high cycle fatigue analysis for cyclic load condition.

\section{Literature Survey}

This review report includes intervention studies on MSDs in all parts of the body. The definition of the health outcomes varies in the original reports from well-defined clinical diagnoses to the reported symptoms of pain or discomfort. In the results of the review, the findings are grouped according to the following anatomical areas

- Low back

- Neck and upper limbs

- Lower limbs.

To be selected, the interventions in the studies had to be targeted on the working system (e.g. ergonomic interventions on the physical environment, tools, methods, work organization) or the mechanisms to handle the related problems at the workplace (e.g. training of workers, operational management of work). Interventions aimed at the treatment of individuals outside the boundaries of the working system are excluded. The scope of the review is the prevention of msd's. Therefore the outcomes of the included studies are related to the health of the musculoskeletal organs with msd's, this classification is problematic because the definition of disorders has usually been based on reported symptoms and there are no medical means to define exactly the onset of diseases related to degeneration (e.g. most of the adults without back pain had anatomic findings of disc degeneration when studied with the latest imaging methods). In the interventions performed at the workplace, it is difficult to exclude people with a history of past pain in order to study mechanisms for primary prevention because the proposed means will obviously also help to prevent the recurrence of pain. Therefore, this review has included studies on both primary and secondary prevention because most of the original studies included all workers within the workplaces investigated. There is also some overlap between secondary and tertiary prevention. Some of the studies included in this report are thus the same as those in the corresponding review being undertaken for the Agency's recent Work related MSDs, only those studies using valid study design are included in the review. Because there are few studies with a randomized setting, the minimum requirement to be included is that there was a comparison group in addition to the intervention group.

\section{Exclusion Criteria}

Case study reports have been excluded from this review. The number of case studies is large and some new experiences are described in the second part of this report. The trials that concentrate mainly on treatment or rehabilitation have also been excluded. They are reviewed in the Work-related MSDs

\section{Low Back}

Organizational and administrative interventions:

Daily working hours were reduced from more than seven hours to six hours in physically demanding care work in three cities in Sweden and Norway Reduced neck and shoulder pain was observed, but not low back pain.

The effects of measures to reduce the physical load in manual handling have been well studied in the laboratory, but the number of field studies with comparison groups is small. In a critical review of 18 such studies by van derMolen seven out of eight studies involving only 


\section{International Journal of Science and Research (IJSR) \\ ISSN (Online): 2319-7064}

Index Copernicus Value (2015): 78.96 | Impact Factor (2015): 6.391

engineering controls (e.g. mechanical aids) found a reduction in physical work demands. Six studies that involved engineering and organizational controls also showed a decrease in workload. Ten studies that reported the effect on MSD symptoms did not show consistent results. One 'high quality' study reported a decline in the incidence of low back disorders. All four of the controlled field studies showed a significant reduction in physical work demands when lifting devices were part of the intervention. Two of these studies measured a significant reduction in low back disorders in the longer term. Other recent studies confirm these findings. Working height adjustment and transport mechanization resulted in lower physical loading and a reduction of back complaints without the loss of productivity in construction work. A new good quality study in the construction industry showed that using a new bricklaying method reduced workload on.

The back and shoulders. The workers were satisfied with the new method, but there was no clear difference in the MSDs between the intervention and comparison groups. A slight decrease in sickness absence was seen in the intervention group. In a health care programme, introducing ergonomic consultation and financial support for purchasing ergonomic devices also resulted in decreased rates of MSDs, although the study had no control group (Fujishiro et al., 2005). There appears to be strong evidence that the introduction of ergonomic improvements may reduce the physical workload

\section{Neck and Upper Limbs}

Disorders of neck and upper limbs are common in many manual tasks and in physically light office work with computers (see below under Office Work). The need for high muscular force in gripping as well as repetitive movements and poor postures have all been found to be work-related risk factors for the development of MSDs in manual work.

\section{Lower Limbs}

Only one study on the prevention of disorders in lower limbs was identified when shock-absorbing and biomechanical shoe or those were tested in military service, users had less back and lower leg disorders than non-users. But because care-seeking for lower extremity problems is rare, the use of this kind of custom-made or those for prevention of MSDs in military conscripts would be too costly for wider application.

\section{Office Work}

MSDs of the neck, shoulders and upper limbs are common among workers using computers. Although the risk of welldefined disease is minor compared with the traditional occupations with repetitive manual tasks, the number of computer users is more than half the workforce in many countries. This results in a very large total number of workers with MSDs. The Directive on computer work aims to reduce the risks. Its recommendations are to adjust the workstation and tools according to the needs of the users, and to train workers to use tools and software properly. The effects of interventions in computerized work have been studied in numerous reports. General reviews on the effectiveness of interventions on MSDs of the neck and upper limbs have also evaluated interventions in an office environment a recent review evaluated over 350 reports related to computer work.

\section{Organizational and administrative interventions}

In physically demanding care work, daily working hours were reduced from more than seven hours to six hours the subjects were compared with workers in similar workplaces who did not benefit from a reduction in working hours. In all intervention groups, the occurrence of neck-shoulder pain was reduced by $15 \%$. No reduction in pain was observed in the reference groups. The prevalence of back pain did not show the same consistent pattern. Extra breaks within the working day have been introduced in some trials, although their long-term effects have not been studied. In a trial in meat-processing plant, the introduction of four nine-minute breaks distributed evenly over the workday for a week were found to reduce the discomfort in the lower limbs but not in other body Areas. The introduction of the breaks did not reduce productivity. In agricultural harvesting, five-minute rest breaks were introduced every working hour and workers in the experimental condition reported significantly less severe symptoms than workers in the control groups.

There are few studies on these interventions. There is limited scientific evidence that a reduction in daily working hours from more than seven hours to six hours can reduce neck and shoulder disorders in physically demanding health care work. There is also evidence that it is possible to introduce additional breaks into repetitive work without loss of productivity. It is not known how the breaks should be organized in order to prevent the occurrence of MSDs most effectively.

There are few studies on these interventions. There is limited scientific evidence that a reduction in daily working hours from more than seven hours to six hours can reduce neck and shoulder disorders in physically demanding health care work. There is also evidence that it is possible to introduce additional breaks into repetitive work without loss of productivity. It is not known how the breaks should be organized in order to prevent the occurrence of MSDs most effectively.

\section{Approach}

\section{Work Activities affecting the neck}

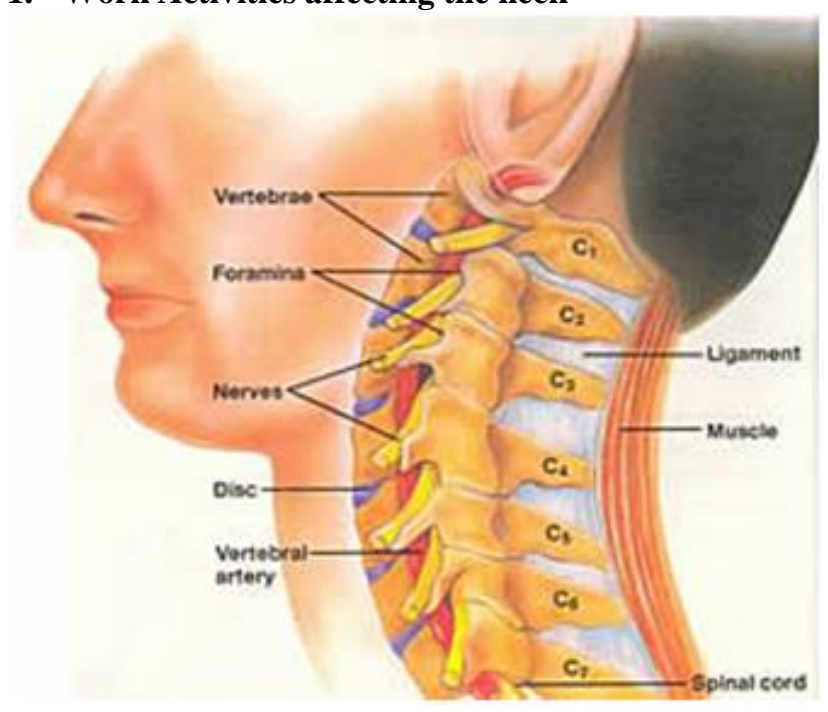


2. Activities affecting the shoulder

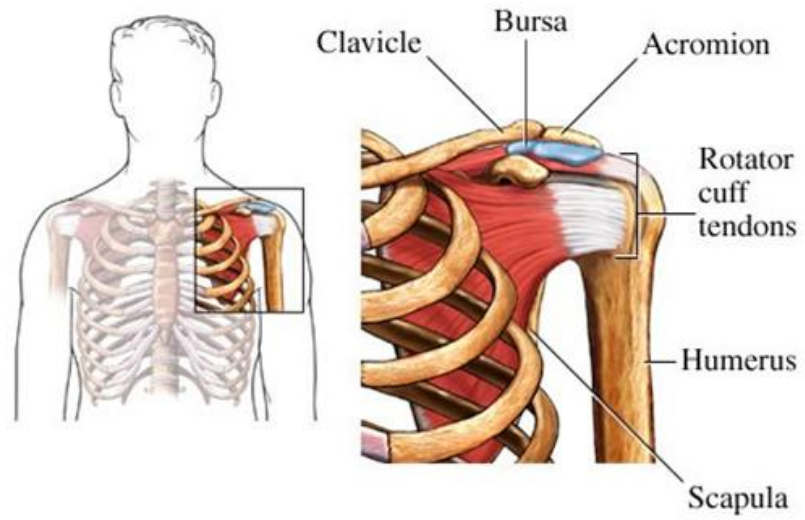

3. Work activities affecting Fingers, Hands and Wrist
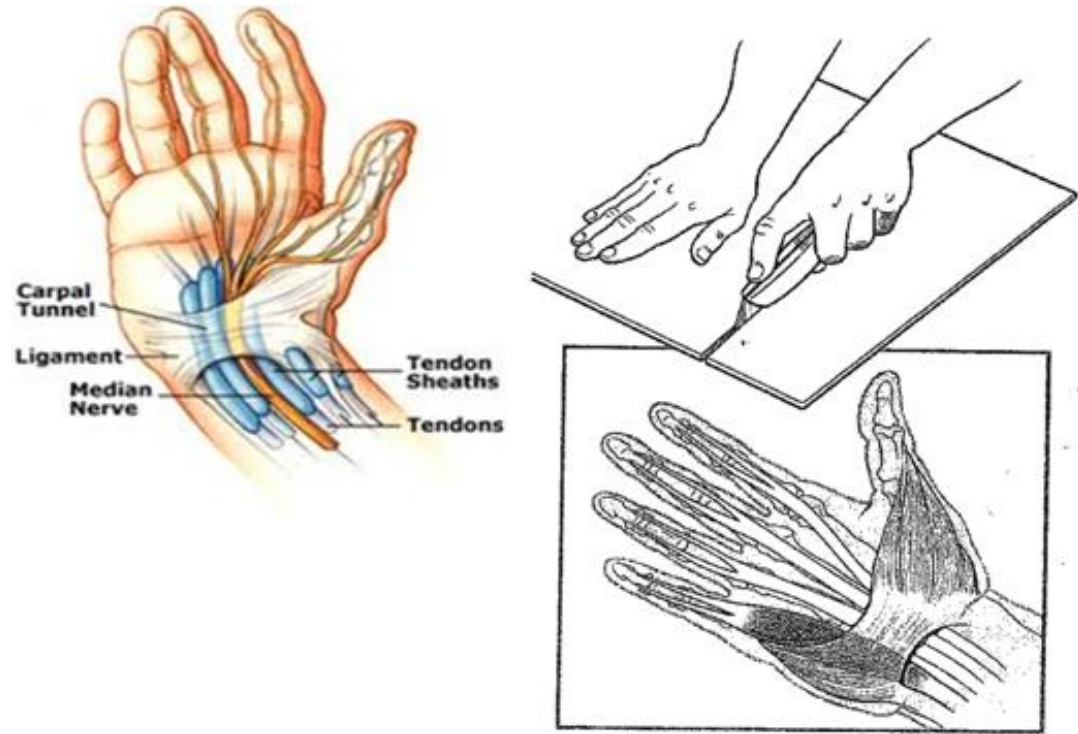

4. Work Activities Affecting the Back
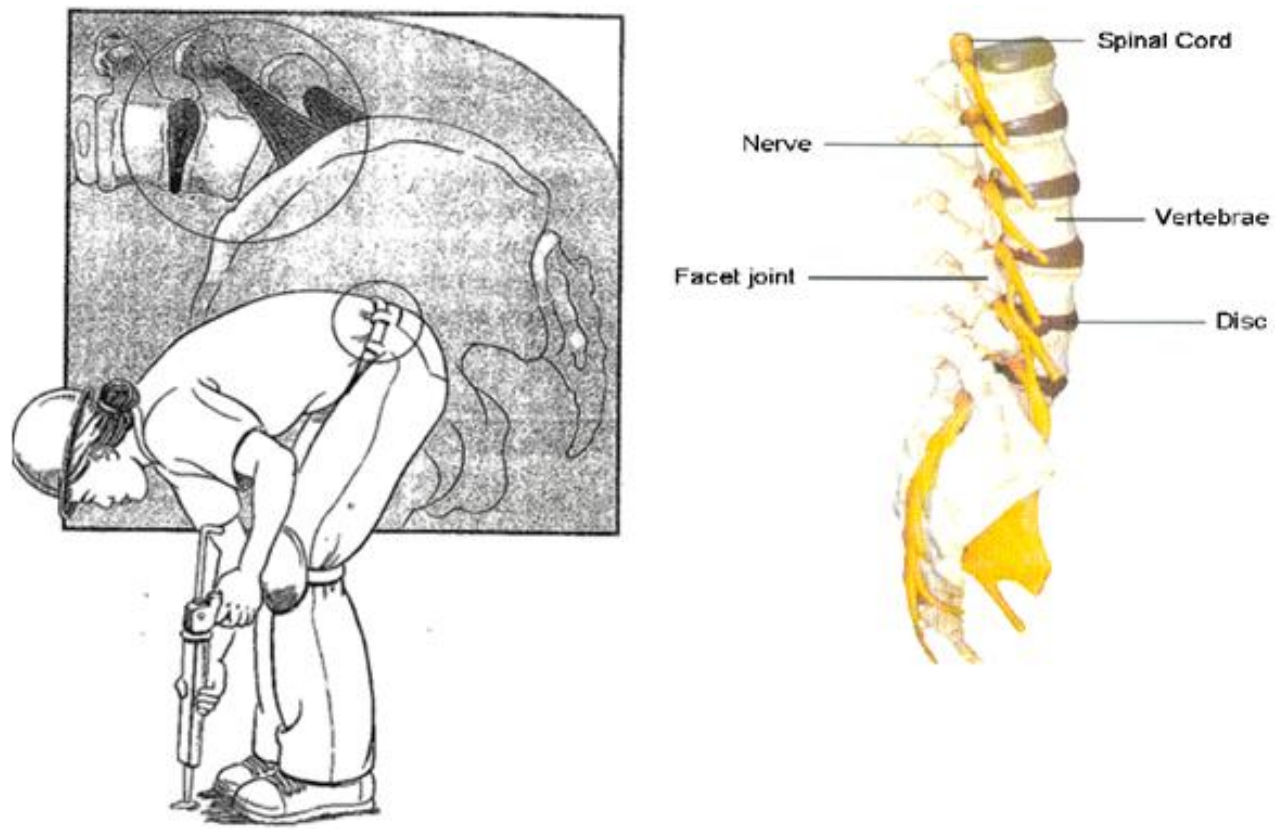

Volume 6 Issue 7, July 2017 www.ijsr.net 


\section{International Journal of Science and Research (IJSR) \\ ISSN (Online): 2319-7064}

Index Copernicus Value (2015): 78.96 | Impact Factor (2015): 6.391

\section{Work Activities Affecting the Knee.}

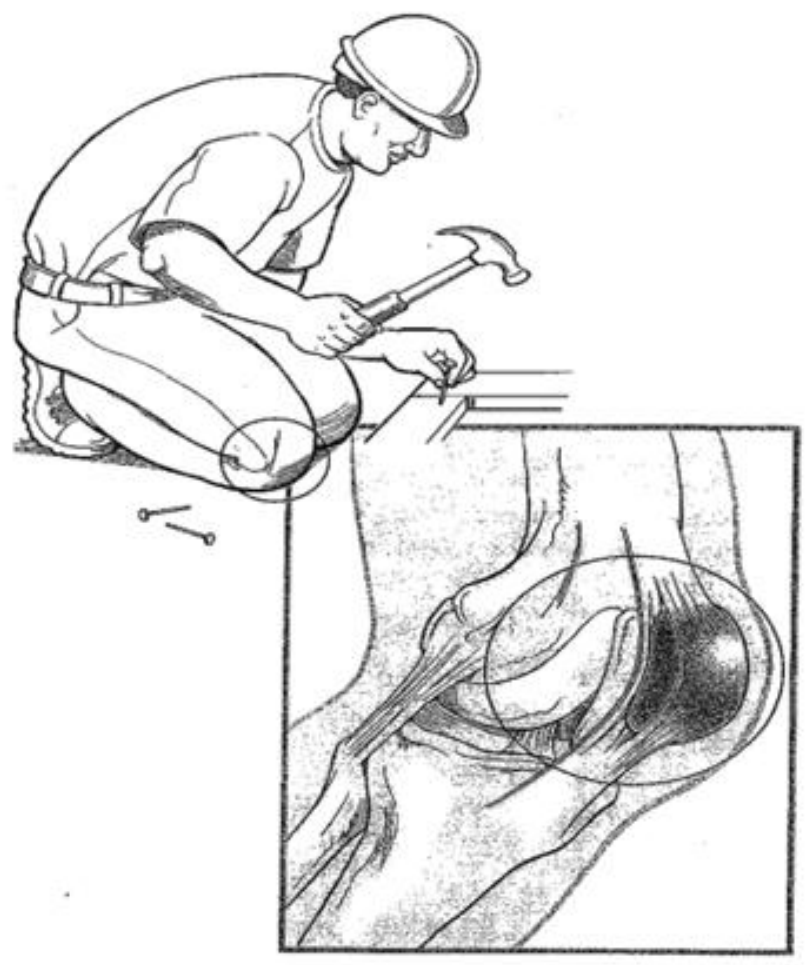

The finite element method (FEM) is a numerical method for solving problems of engineering and mathematical physics. It is also referred to as finite element analysis (FEA), to solve the problem it subdivides a large problem into smaller, simpler parts that are called finite elements.

FEM then uses variation methods from the calculus of variations to approximate a solution by minimizing an associated error function.

The subdivision of a whole domain into simpler parts has several advantages:

- Accurate representation of complex geometry.

- Inclusion of dissimilar material properties.

- Easy representation of the total solution.

- Capture of local effects.

- EM is best understood from its practical application, known as finite element analysis .(FEA)

- FEA as applied in engineering is a computational tool for performing engineering analysis.

Finite element analysis is a good choice for analyzing problems over complicated domains when the domain changes (as during a solid state reaction with a moving boundary), Finite element analysis is used to find the behaviour of the structure under the loading condition, and the perfection in model is one of the factors influencing the accuracy of the solution.

\section{Pre-processor}

Pre-processor involves the choosing the materials that are involved in the analysis and entering the material data which are missing the engineering data. Meshing the model is another important process in the Finite Element Analysis, where certain condition has to be followed like aspect ratio and skew angle. Assignment of the material to the structure and parts, in case if the different part has different material. The predetermined boundary condition and the loading condition are imposed on the Finite Element Model.

\section{Solver}

Solver stage is fully automatic. It is mainly divided into presolver and post-solver. Pre-solver reads the model created by pre-processor and formulates the model in the mathematical representation. All the pre-processor process must be satisfied to create the mathematical model, and then the mathematical engine proceeds to form the elemental stiffness matrix for the given problem. Solver engine solves the problem and calculates to give the required result like stress, strain, displacement, temperature and frequency etc.

\section{Post-processor}

Post processing procedures are designed for the extraction of the data of interest from a finite element solution. In order to meet the requirements of solution verification, postprocessors need to provide for a posteriori error estimation in terms of the quantities of interest. In postprocessor results obtained by the solver is read and interpreted. The results are interpreted in terms of deformation, mode shapes and natural frequency by the animation and the graph. Stress obtained are analysed by the stress plot which used know the maximum and minimum stress is present and by this we can conclude, weather the design is safe or not. Design modification can carried out in those areas where the attention is required. It gives the representation of the stress, strain and deflection in any direction or in required direction also at any angle to the coordinate axis.

Finite Elements Method encompasses methods for connecting many simple element equations over many small subdomains, named finite elements, to approximate a more complex equation over a larger domain.

Linear Static Analysis : It is the simplest and most commonly used type of analysis

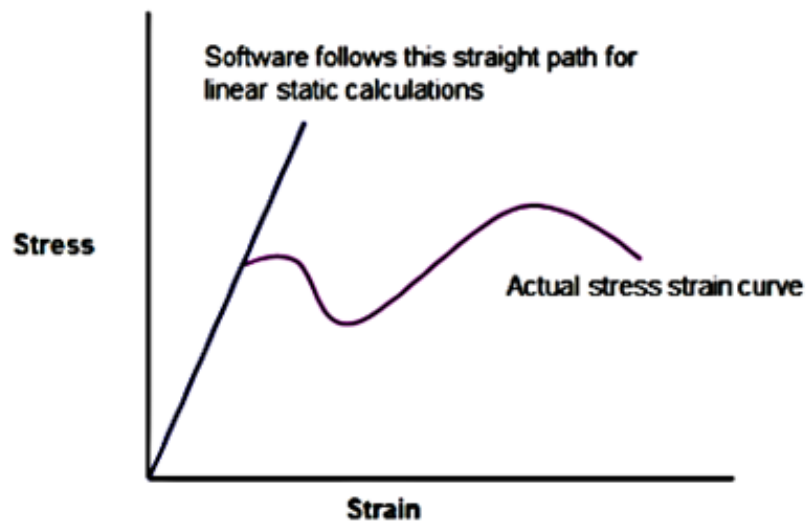

Linear means straight line. $\sigma=€ \mathrm{E}$ is the equation of straight line $(y=m x+c)$ passing through origin. 'E', Young's modulus is the slope of curve and is a constant. In real life, after passing yield point material follows nonlinear curve but software follows same straight line. Component break into two separate pieces after crossing ultimate stress but software based analysis never show failure in this fashion. It 


\section{International Journal of Science and Research (IJSR) \\ ISSN (Online): 2319-7064 \\ Index Copernicus Value (2015): 78.96 | Impact Factor (2015): 6.391}

shows single unbroken part only with red color zone at the location of failure. Analyst has to conclude whether the component is safe or fail by comparing maximum stress value with the yield or ultimate stress.

There are two conditions for static analysis:

No variation of force with respect to time (Dead weight)

Equilibrium condition $-\sum$ Force $=0$ and $\sum$ Moments $=0$.

Hence, peak linear stress linear strain will be calculated by this method for dead frontal axle under defined loading conditions.

\section{Bilinear Kinematic Hardening}

Hardening : The yield criterion for many materials depends on the history of loading and evolution of plastic strain. The change in the yield criterion due to loading is called hardening and is defined by the hardening rule. Hardening behaviour results in an increase in yield stress upon further loading from a state on the yield surface so that for a plastically deforming material, an increase in stress is accompanied by an increase in plastic strain.

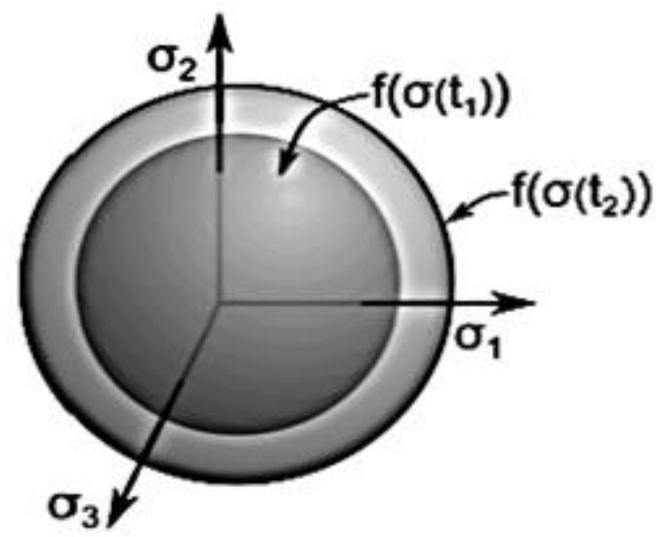

Two common types of hardening rules are isotropic and kinematic hardening. Isotropic type of hardening can model the behavior of materials under monotonic loading and elastic unloading, but often does not give good results for structures that experience plastic deformation after a load reversal from a plastic state.

Kinematic hardening is observed in cyclic loading of metals. It can be used to model behavior such as the Bauschinger effect, where the compressive yield strength reduces in response to tensile yielding.

It can also be used to model plastic ratcheting, which is the build-up of plastic strain during cyclic loading.

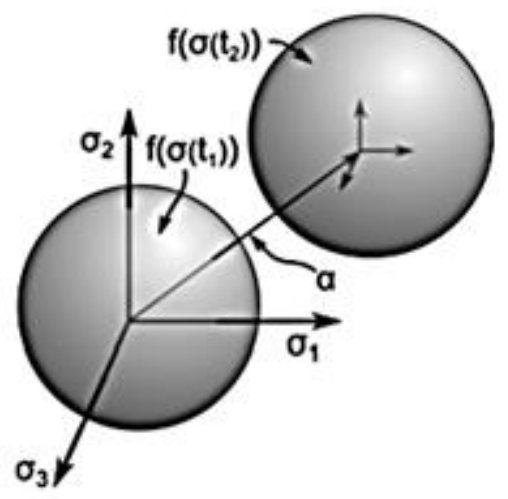

Since the considered dead frontal axle is subjected to cyclic loading and the yielding surface cross section remains constant, Bilinear Kinematic Hardening method is use for analysis

\section{Kinematic Hardening}

During plastic deformation, kinematic hardening causes a change in the yield stress. In uniaxial tension, plastic deformation causes the tensile yield stress to increase and the magnitude of the compressive yield stress to decrease. This type of hardening can model the behaviour of materials under either monotonic or cyclic loading and can be used to model phenomena such as the Bauschinger effect and plastic ratcheting.

The yield criterion has the form $\mathrm{F}\left(\sigma^{\prime}\right)-\sigma \mathrm{y}=0$

Where $\mathrm{F}\left(\sigma^{\prime}\right)$ is a scalar function of the relative stress $\sigma^{\prime}$ and $\sigma y$ is the yield stress.

The relative stress is, $\sigma^{\prime}=\sigma-\alpha$

Where the backstress $\alpha$ is the change in the position of the yield surface in stress space and evolves during plastic deformation

\section{Bilinear Kinematic Hardening}

The backstress tensor for bilinear kinematic hardening evolves so that the effective stress versus effective strain curve is bilinear.

The initial slope of the curve is the elastic modulus of the material and beyond the user specified initial yield stress $\sigma_{0}$, plastic strain develops and the back stress evolves so that stress versus total strain continues along a line with slope defined by the user specified tangent modulus ET.

This tangent modulus cannot be less than zero or greater than the elastic modulus. For uniaxial tension followed by uniaxial compression, the magnitude of the compressive yield stress decreases as the tensile yield stress increases so that the magnitude of the elastic range is always $2 \sigma 0$,

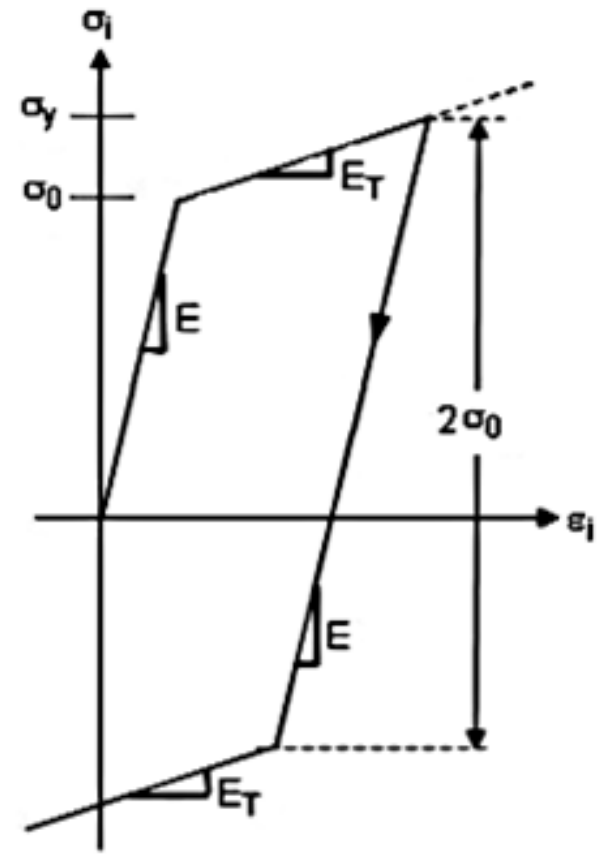

Figure: Stress v/s Total Strain for Bilinear Kinematic Hardening 


\section{International Journal of Science and Research (IJSR) \\ ISSN (Online): 2319-7064 \\ Index Copernicus Value (2015): 78.96 | Impact Factor (2015): 6.391}

In this method yield stress, tangent modulus and young's modulus for the dead frontal axle model will be provided through commercial FE package to find out the true stress and true strain. The true strain obtained by this method will be a combination of elastic strain and plastic strain.

\section{Neuber's Rule}

Neuber showed that for a shear-strained prismatic body with an random non-linear stress-strain curve, the geometric mean of the stress and strain concentration factors (Ko and $\mathrm{K} €)$ is equal to the theoretical stress concentration factor $\mathrm{Kt}$.

This is expressed as follows:

Where,

$$
K_{\sigma} K_{\epsilon}=K \tau^{2}
$$

$\mathrm{K} \sigma=$ actual stress concentration factor

$\mathrm{K} €=$ actual strain concentration factor

$\mathrm{Kt}=$ stress concentration factor for a linear elastic material

The stress concentration factor, $\mathrm{K} \sigma$ is the ratio of the notch root stress and the net-section nominal stress.

$$
K_{\sigma}=\frac{\sigma}{S}
$$

The strain concentration factor, $\mathrm{K} €$ is the ratio of the notch root strain and the net-section nominal strain e.

$$
K_{\varepsilon}=\frac{\varepsilon}{e}
$$

Hence, Neuber's rule is re-written as:

$$
\sigma_{\varepsilon}=K_{t}^{2} S e
$$

Generally, this is re-written in terms of stress and strain ranges for the case when the stress range remote to the notch is linear elastic:

$$
\Delta \sigma \Delta \varepsilon=\frac{\left(\mathrm{K}_{\mathrm{t}} \Delta \mathrm{S}\right)^{2}}{\mathrm{E}}
$$

The form presented in the above equation is widely used in fatigue life calculations using the linear static approach. It shows that the product of the notch stress and strain ranges can be estimated by knowing the theoretical stress concentration factor, the applied stress range and the elastic modulus of the material.

\section{Conclusion}

The resulted values of linear static analysis will be substituted in Neuber's rule to determine the true stress and true strain. The obtained results of this analytical method will be compared with the output of Bilinear Kinematic Hardening method. Thus establishing a correlation between both the methods, a correction factor will be found out and a customized method of evaluation is developed leading to reduced overall design cycle of the part.

\section{References}

[1] Ammendolia, C., Kerr, M.S., et al., 'Back belt use for prevention of occupational low back pain: a systematic review', Journal of Manipulative and Physiological Therapeutics, 28(2), 2005, pp. 128-134.

[2] Bos, E.H., Krol, B., et al., 'The effects of occupational interventions on reduction of musculoskeletal symptoms in the nursing profession', Ergonomics, 49(7), 2006, pp. 706-723.

[3] Brewer, S., Eerd, D.V., et al., 'Workplace interventions to prevent musculoskeletal and visual symptoms and disorders among computer users: a systematic review', Journal of Occupational Rehabilitation, 16(3), 2006, pp. 317-350.

[4] Buckle, P., Devereux, J., European Agency for Safety and Health at Work, Work-related neck and upper limb musculoskeletal disorders, Luxembourg, Office for Official Publications of the European Communities, 1999,

at: http://osha.eu.int/publications/reports/201/en/index.htm

[5] Dababneh, A.J., Swanson, N., et al., 'Impact of added rest breaks on the productivity and well-being of workers', Ergonomics, 44(2), 2001, pp. 164-174. De Beeck, R.O., Hermans, V., European Agency for Safety and Health at Work, Research on work-related low back disorders, Luxembourg, Office for Official Publications of the European Communities, 2000, at: http://osha.eu.int/publications/reports/204/en/index.htm

[6] Dishman, R.K., Oldenburg, B., et al., 'Worksite physical activity interventions', American Journal of Preventative Medicine, 15(4), 1998, pp. 344-361. Engbers, L.H., van Poppel, M.N., et al., 'Worksite health promotion programmes with environmental changes: a systematic review', American Journal of Preventative Medicine, 29(1), 2005,

[7] European Agency for Safety and Health at Work, Workrelated MSDs: back-to-work report, Luxembourg: Office for Official Publications of the European Communities, 2007. European Commission, Adapting to change in work and society: a new Community strategy on health and safety at work 2002-2006, COM (2002)118, Brussels, European Commission, 2002.

[8] European Commission, Improving quality and productivity at work: Community strategy 2007-2012 on health and safety at work, COM (2007)62, Brussels, European Commission, 2007.

[9] European Foundation for the Improvement of Living and Working Conditions, Fourth European Working Conditions Survey, Luxembourg: Office for Official Publications.

\section{Author Profile}

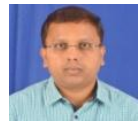

K .V. Manjunath, Received the B.E and M.E degrees in Mechanical Engineering from SIT-Tumkur and UVCE Bangalore, 1999 and 2004 respectively from Bangalore University Karnataka. He is having nearly 15 years of teaching experience. He is working as Assistant Professor at SIT Tumkur. Now he is doing research in the area of role of ergonomics on msd's.

Dr. B. Ravishankar, Received the B.E and M.E. and $\mathrm{Ph} \mathrm{D}$ degrees in 1990, 1992, and 2003 respectively in Mechanical Engineering from Bangalore University, Karnataka. He is working as Professor (IEM) \& Placement Officer, BMS College of Engineering, Bangalore-19, Karnataka. He is having nearly 25 years of teaching experience, his area of research is supply chain management. Ergonomics, Simulation and ERP. 